\title{
Decidability of Weak Bisimilarity for a Subset of Basic Parallel Processes
}

\author{
Colin Stirling \\ Division of Informatics \\ University of Edinburgh \\ email: cps@dcs.ed.ac.uk
}

\section{Introduction}

In the past decade there has been a variety of results showing decidability of bisimulation equivalence between infinite state systems. The initial result, due to Baeten, Bergstra and Klop [1], proved decidability for normed BPA processes, described using irredundant context-free grammars. This was extended to all BPA processes and then to pushdown automata 51614]. Decidability of bisimilarity was also shown for Basic Parallel (BP) processes, a restricted subset of Petri nets, 4]. For full Petri nets Jančar proved that bisimulation equivalence is undecidable [11].

An open question is the dividing line between decidability and undecidability of bisimilarity in the case of "sequential" systems. For instance, is bisimulation equivalence decidable for the general class of prefix-recognisable transition graphs introduced by Caucal [2]? A poignant problem is that these graphs exhibit infinite branching. Families of infinite state systems for which bisimilarity is known to be decidable are finitely branching. For each label $a$ and for each configuration the set of its $a$-successors is finite and easily computable. Therefore if two systems are not bisimulation equivalent then there is a least approximant $n>0$ such that they are not equivalent at level $n$, and for each $n$ the equivalence at level $n$ is decidable. But if processes are infinite branching then inequivalence may be manifested at higher ordinals, and therefore a new technique is required to establish semidecidability of inequivalence.

Instead of examining richer families of infinite state systems one can look at the problem of deciding weak bisimulation equivalence for restricted classes. Weak bisimilarity abstracts from silent activity, with the consequence that BPA and BP processes are infinitely branching. Weak bisimulation inequivalence is then generally not finitely approximable.

In this paper we examine the decision problem of weak bisimilarity for normed BP processes. Esparza [6] observes that weak bisimilarity is semidecidable, because a positive witness is semilinear. Decidability was proved for a restricted subclass, the totally normed processes, by Hirshfeld [7]. And Jančar, Kučera and Mayr show decidability of weak bisimilarity between general (PA) processes which includes BP processes and finite state processes [12]. However in both these cases inequivalence is finitely approximable. In this paper we prove decidability 
of weak bisimilarity for a subset of normed BP processes for which inequivalence need not be finitely approximable. Underpinning this result is a finite symbolic characterisation of the infinite branching of normed BP processes. Indeed we believe that the technique will establish decidability of weak bisimilarity for all normed BP processes, but the combinatorics become awesome.

In section 2 we define normed Basic Parallel processes and weak bisimulation equivalence. Section 3 is devoted to the finite characterisation of the infinite transition relations. Then in section 4 we utilise the characterisation to prove the decidability result using the tableau method. Proofs of two crucial lemmas are given in section 5 .

\section{Normed Basic Parallel Processes}

Ingredients of Basic Parallel (BP) processes are a finite set $\Gamma=\left\{X_{1}, \ldots, X_{n}\right\}$ of atoms, a finite set $\mathrm{A}=\left\{a_{1}, \ldots, a_{k}\right\}$ of actions and a finite set $\mathrm{T}$ of basic transitions, each of the form $X \stackrel{a}{\longrightarrow} \alpha$ where $X$ is an atom, $a \in \mathrm{A} \cup\{\tau\}$ and $\alpha$ is a multiset of atoms whose size is at most 2. A BP process, or configuration, is a parallel composition of atoms. We let $\alpha, \beta, \ldots$ range over such processes. A process therefore has the form $X_{1}^{k_{1}} \ldots X_{n}^{k_{n}}$, which is the parallel composition of $k_{1}$ copies of $X_{1}, \ldots$ and $k_{n}$ copies of $X_{n}$ where each $k_{i} \geq 0$. We let $\epsilon$ be the empty composition, where each $k_{i}=0$. If $\alpha$ and $\beta$ are two processes then $\alpha \beta$ is their multiset union (and we often write $X \alpha$ or $\alpha X$ as an abbreviation for the multiset union of $\{X\}$ and $\alpha$ ). The behaviour of a BP process is determined by the following extension rule: if $X \stackrel{a}{\longrightarrow} \alpha \in \mathrm{T}$ then $X \beta \stackrel{a}{\longrightarrow} \alpha \beta$. The silent action $\tau \notin \mathrm{A}$ is included as a possible action. We assume the usual expansion of the transition relation to words, $\alpha \stackrel{w}{\longrightarrow} \beta$ where $w \in(\mathrm{A} \cup\{\tau\})^{*}$.

Example 1 The atoms $\Gamma$ are $\{A, Y, Z\}$ and $\mathrm{A}$ is the singleton set $\{a\}$. The basic transitions are $A \stackrel{a}{\longrightarrow} \epsilon, Y \stackrel{a}{\longrightarrow} A, Z \stackrel{a}{\longrightarrow} A, Y \stackrel{\tau}{\longrightarrow} Y A, Z \stackrel{a}{\longrightarrow} Z, Z \stackrel{\tau}{\longrightarrow} Z A$. $Z A^{3}$ has the following transitions, $Z A^{3} \stackrel{a}{\longrightarrow} A^{4}, Z A^{3} \stackrel{a}{\longrightarrow} Z A^{2}, Z A^{3} \stackrel{a}{\longrightarrow} Z A^{3}$ and $Z A^{3} \stackrel{\tau}{\longrightarrow} Z A^{4}$.

Example 2 The atoms are $\{C, D, U, V\}, \mathrm{A}=\{c, d\}$ and the basic transitions are $U \stackrel{\tau}{\longrightarrow} U D, U \stackrel{c}{\longrightarrow} \epsilon, U \stackrel{c}{\longrightarrow} C, U \stackrel{{ }^{c}}{\longrightarrow} U, V \stackrel{\tau}{\longrightarrow} V D, V \stackrel{c}{\longrightarrow} \epsilon, V \stackrel{c}{\longrightarrow} C$, $C \stackrel{c}{\longrightarrow} C, C \stackrel{c}{\longrightarrow} \epsilon, D \stackrel{d}{\longrightarrow} \epsilon, D \stackrel{\tau}{\longrightarrow} \epsilon$. For each $n \geq 0$ there is the extended transition $U \stackrel{\tau^{n} c}{\longrightarrow} U D^{n}$.

$\mathrm{BP}$ processes are communication free Petri nets, where the places are the atoms, and transition $X \stackrel{a}{\longrightarrow} \alpha$ is a firing rule. A configuration $X_{1}^{k_{1}} \ldots X_{n}^{k_{n}}$ represents the marking when there are $k_{i}$ tokens on place $X_{i}$. They are communication free because each transition requires just one place to fire. As is usual in process calculi when there are silent transitions, the weak transition relations $\stackrel{\epsilon}{\Longrightarrow}$ and $\stackrel{a}{\Longrightarrow}$ for $a \in \mathrm{A}$ are defined as follows.

$$
\alpha \stackrel{\epsilon}{\Longrightarrow} \beta \text { iff } \exists n \geq 0 . \alpha \stackrel{\tau^{n}}{\longrightarrow} \beta \quad \alpha \stackrel{a}{\Longrightarrow} \beta \text { iff } \exists \alpha_{1}, \beta_{1} . \alpha \stackrel{\epsilon}{\Longrightarrow} \alpha_{1} \stackrel{a}{\longrightarrow} \beta_{1} \stackrel{\epsilon}{\Longrightarrow} \beta
$$


There can be infinite branching with respect to these transition relations, as illustrated by Examples 1 and $2, Y \stackrel{\epsilon}{\Longrightarrow} Y A^{n}$ and $V \stackrel{c}{\Longrightarrow} C D^{n}$ for all $n \geq 0$.

An atom $X$ is normed if there is a word $w \in \mathrm{A}^{*}$ such that $X \stackrel{w}{\Longrightarrow} \epsilon$. A BP definition is normed if all its atoms are normed. Both Examples 1 and 2 are normed. The norm of atom $X$, written $\mathrm{N}(X)$, is the length of a shortest word $w$ such that $X \stackrel{w}{\Longrightarrow} \epsilon . \mathrm{N}(Y)=2$ and $\mathrm{N}(D)=0$ where $Y$ and $D$ are from the examples above. Norm extends to configurations $\alpha$, written $\mathrm{N}(\alpha)$, which is the length of a shortest word $w$ such that $\alpha \stackrel{w}{\Longrightarrow} \epsilon$. If a BP definition is normed then so is any process $\alpha=X_{1}^{k_{1}} \ldots X_{n}^{k_{n}}$ and $\mathrm{N}(\alpha)$ is $\sum_{1 \leq i \leq n}\left(k_{i} \times \mathrm{N}\left(X_{i}\right)\right)$. A subset of normed BP processes is the totally normed processes, as introduced by Hüttel and examined by Hirshfeld 97. A BP process definition is totally normed if all its atoms are normed and have norm greater than 0. Example 1 is totally normed but Example 2 is not because $\mathrm{N}(D)=0$.

Our interest is with deciding when two normed BP processes are weak bisimulation equivalent. There is more than one way to define this equivalence. First we start with the natural (and "symmetric") version.

Definition 1 A binary relation $B$ between BP processes is a weak bisimulation relation provided that whenever $\alpha B \beta$ and $a \in(\mathrm{A} \cup\{\epsilon\})$

if $\alpha \stackrel{a}{\Longrightarrow} \alpha^{\prime}$ then there is a $\beta^{\prime}$ such that $\beta \stackrel{a}{\Longrightarrow} \beta^{\prime}$ and $\alpha^{\prime} B \beta^{\prime}$

if $\beta \stackrel{a}{\Longrightarrow} \beta^{\prime}$ then there is an $\alpha^{\prime}$ such that $\alpha \stackrel{a}{\Longrightarrow} \alpha^{\prime}$ and $\alpha^{\prime} B \beta^{\prime}$

Two processes $\alpha$ and $\beta$ are weakly bisimilar, written $\alpha \approx \beta$, if there is a weak bisimulation relation $B$ such that $\alpha B \beta$. Important properties of weak equivalence are that it is a congruence for BP processes, see [7] for instance, and that it preserves norm.

Fact 1 If $\alpha \approx \beta$ then $\alpha \delta \approx \beta \delta$ and $\mathrm{N}(\alpha)=\mathrm{N}(\beta)$.

An alternative (and equivalent) basis for the definition of weak bisimilarity is as follows, where if $a \in A$ then $\hat{a}$ is $a$ and if $a=\tau$ then $\hat{a}$ is $\epsilon,[13$.

Definition 2 A binary relation $B$ between BP processes is a wb relation provided that whenever $\alpha B \beta$ and $a \in(\mathrm{A} \cup\{\tau\})$

if $\alpha \stackrel{a}{\longrightarrow} \alpha^{\prime}$ then there is a $\beta^{\prime}$ such that $\beta \stackrel{\hat{a}}{\Longrightarrow} \beta^{\prime}$ and $\alpha^{\prime} B \beta^{\prime}$

if $\beta \stackrel{a}{\longrightarrow} \beta^{\prime}$ then there is an $\alpha^{\prime}$ such that $\alpha \stackrel{\hat{a}}{\Longrightarrow} \alpha^{\prime}$ and $\alpha^{\prime} B \beta^{\prime}$

Fact $2 B$ is a wb relation iff $B$ is a weak bisimulation relation.

To establish that $\alpha \approx \beta$ it therefore suffices to exhibit a binary relation containing $\alpha$ and $\beta$ and prove that it is a wb relation. In general such a relation will be infinite. The relation $\left\{\left(Y A^{n}, Z A^{n}\right),\left(A^{n}, A^{n}\right): n \geq 0\right\}$ over processes of Example 1 is a wb relation, which proves that $Y \approx Z$.

The "symmetric" definition of equivalence supports weak bisimulation approximants, $\approx_{o}$ for any ordinal $o$, which are themselves equivalence relations.

Definition 3 The relations $\approx_{o}$ for ordinals $o$ are defined inductively as follows, where we assume that $l$ is a limit ordinal ( $\operatorname{such}$ as $\omega$ ). 


$$
\begin{aligned}
& \alpha \approx_{0} \beta \\
& \alpha \approx_{o+1} \beta \text { iff for } a \in(\mathrm{A} \cup\{\epsilon\}) \\
& \quad \text { if } \alpha \stackrel{a}{\Longrightarrow} \alpha^{\prime} \text { then } \exists \beta^{\prime} . \beta \stackrel{a}{\Longrightarrow} \beta^{\prime} \text { and } \alpha^{\prime} \approx_{o} \beta^{\prime} \\
& \quad \text { if } \beta \stackrel{a}{\Longrightarrow} \beta^{\prime} \text { then } \exists \alpha^{\prime} . \alpha \stackrel{a}{\Longrightarrow} \alpha^{\prime} \text { and } \alpha^{\prime} \approx_{o} \beta^{\prime} \\
& \alpha \approx_{l} \beta \text { iff } \forall o<l . \alpha \approx_{o} \beta
\end{aligned}
$$

Fact $3 \alpha \approx \beta$ iff for all ordinals $o . \alpha \approx_{o} \beta$.

Example 2 illustrates the need for ordinals beyond $\omega$. Although $U \neq V$ for any $n \geq 0, U \approx_{n} V$. The inequivalence is due to the transition $U \stackrel{c}{\Longrightarrow} U$. Process $V$ does not have a similar transition. However for any $n \geq 0, V \stackrel{c}{\Longrightarrow} C D^{n}$ and $U \approx_{n} C D^{n}$ but $U \not_{n+1} C D^{n}$ because of the transition $U \stackrel{d}{\Longrightarrow} U D^{n}$. Therefore it follows that $U \not_{\omega+1} V$. It is conjectured by Strríbrná [15] that one only needs ordinals which are less than $\omega \times 2$ to establish inequivalence between all BP processes, including the unnormed. She proves this in the special case when there are no atoms of norm 0 and $A$ is a singleton set. And in the case of totally normed processes she shows that the closure ordinal is $\omega$ : if $\alpha \not \approx \beta$ then for some $n \geq 0, \alpha \neq_{n} \beta$.

Esparza observes that a wb relation which witnesses the equivalence $\alpha \approx \beta$ is semilinear [6], which establishes semidecidability of weak bisimilarity for all BP processes (and therefore decidability for totally normed processes). But the problem is establishing semidecidability of inequivalence.

A new approach to deciding weak equivalence is now developed. First we finitely characterise the infinite branching of a normed BP process, and then we use the characterisation to show that equivalence and inequivalence can be captured by examining only boundedly many transitions. However we are only able to prove decidability for a subset of normed BP processes which includes the totally normed processes. The family also includes Example 2 where inequivalence is not finitely approximable. The subset is given by a technical restriction, whose notation is now developed.

\section{Stratification and Generators}

In this section we symbolically characterise the weak transition relations of normed BP processes. Assume a fixed normed BP process definition with atoms $\Gamma$, action set $A$ and transitions $\mathrm{T}$. The initial step is to stratify the basic transitions in $\mathrm{T}$, by including a numerical index on the transition relation which represents the change in norm produced by the transition. If $X \stackrel{a}{\longrightarrow} \alpha \in \mathrm{T}$ then we re-write it as $X \stackrel{a}{\longrightarrow} n$ where $n=\mathrm{N}(\alpha)-\mathrm{N}(X)$. The index $n$ is bounded, $-1 \leq n \leq 2 \mathrm{M}$, where $\mathrm{M}$ is the maximum norm of any atom in $\Gamma$. Either a transition is norm reducing, but then by at most 1 , or it is nondecreasing and because $|\alpha| \leq 2$ the increase in norm is at most $2 \mathrm{M}$. An important, but simple, observation is that for a stratified $\tau$-transition, $X \stackrel{\tau}{\longrightarrow}$, $\alpha$, the index $n$ must be nondecreasing, $n \geq 0$. A selection of stratified transitions from Examples 1 and 2 of the previous section is $A \stackrel{a}{\longrightarrow}{ }_{-1} \epsilon, Z \stackrel{a}{\longrightarrow} Z, Y \stackrel{\tau}{\longrightarrow} Y A, U \stackrel{c}{\longrightarrow}{ }_{-1} \epsilon$, $D \stackrel{\tau}{\longrightarrow} 0, V \stackrel{\tau}{\longrightarrow}$ VD. 
The definition of stratification is extended to the weak transition relations as follows.

$$
\begin{aligned}
& \alpha \stackrel{\epsilon}{\Longrightarrow}{ }_{0} \beta \quad \text { iff } \exists m>0 . \exists \alpha_{1}, \ldots, \alpha_{m} . \alpha=\alpha_{1} \stackrel{\tau}{\longrightarrow} 0 \ldots \stackrel{\tau}{\longrightarrow}{ }_{0} \alpha_{m}=\beta \\
& \alpha \stackrel{\epsilon}{\Longrightarrow} n+1 \beta \text { iff } \exists \alpha^{\prime}, \beta^{\prime} . \alpha \stackrel{\epsilon}{\Longrightarrow}_{j} \alpha^{\prime} \stackrel{\tau}{\longrightarrow}_{k+1} \beta^{\prime} \stackrel{\epsilon}{\Longrightarrow}_{l} \beta \quad \text { where } n=j+k+l \\
& \alpha \stackrel{a}{\Longrightarrow} n \text { iff } \exists \alpha^{\prime}, \beta^{\prime} . \alpha \stackrel{\epsilon}{\Longrightarrow}_{j} \alpha^{\prime} \stackrel{a}{\longrightarrow}_{k} \beta^{\prime} \stackrel{\epsilon}{\Longrightarrow}_{l} \beta \quad \text { where } n=j+k+l
\end{aligned}
$$

For instance, $U \stackrel{\epsilon}{\Longrightarrow}{ }_{0} U D^{64}$ and $U \stackrel{c}{\Longrightarrow}{ }_{-1} D^{80}$ are stratified weak transitions arising from Example 2 of the previous section.

Weak bisimulation equivalence can be redefined using the stratified weak transition relations. Assume that $\mathrm{K}$ is the largest increase in norm of the $\mathrm{BP}$ process definition, $\max \left\{n: X \stackrel{a}{\longrightarrow}_{n} \alpha \in \mathrm{T}\right\}$. For instance, $\mathrm{K}=1$ for Example 1 of the previous section.

Definition 1 A relation $B$ between normed BP processes is a stratified weak bisimulation relation provided that whenever $\alpha B \beta$ and $n \leq \mathrm{K}$ and $a \in(\mathrm{A} \cup\{\epsilon\})$

if $\alpha \stackrel{a}{\Longrightarrow}{ }_{n} \alpha^{\prime}$ then there is a $\beta^{\prime}$ such that $\beta \stackrel{a}{\Longrightarrow} n \beta^{\prime}$ and $\alpha^{\prime} B \beta^{\prime}$ if $\beta \stackrel{a}{\Longrightarrow} \beta^{\prime}$ then there is an $\alpha^{\prime}$ such that $\alpha \stackrel{a}{\Longrightarrow}{ }_{n} \alpha^{\prime}$ and $\alpha^{\prime} B \beta^{\prime}$

Proposition $1 B$ is a stratified weak bisimulation relation iff $B$ is a wb relation.

Hence $\alpha \approx \beta$ iff there is a stratified weak bisimulation relation $B$ which contains the pair $\alpha$ and $\beta$. In the next section we shall also define associated stratified weak approximants.

The crucial feature of totally normed processes is that they are finitely branching with respect to the stratified weak transition relations.

Fact 1 If $\alpha$ is totally normed then for all $a$ and $n\left\{\delta: \alpha \stackrel{a}{\Longrightarrow}{ }_{n} \delta\right\}$ is finite.

This is not generally the case for normed BP processes. For instance $V \stackrel{c}{\Longrightarrow}{ }_{-1} D^{n}$ for all $n \geq 0$. The crucial component of infinite branching is the relation $\stackrel{\epsilon}{\Longrightarrow}$, to which we now direct our analysis. The following result is useful.

Proposition 2 If $\alpha \stackrel{\epsilon}{=} \beta$ and $\beta \stackrel{\epsilon}{=} \delta$ and $\alpha \approx \delta$ then $\alpha \approx \beta$.

Consequently if $X \stackrel{\epsilon}{\Longrightarrow} Y$ and $Y \stackrel{\epsilon}{\Longrightarrow}{ }_{0} X$ then $X \approx Y$. In this circumstance, if $X \neq Y$ then we say that atom $Y$ is redundant because of $X$. A BP definition can therefore be replaced with an equivalent definition which does not contain redundant atoms. If $Y \in \Gamma$ is redundant because of $X \in \Gamma$ then we change $\Gamma$ to $\Gamma-\{Y\}$ and we replace all transitions $Y \stackrel{a}{\longrightarrow} \alpha \in \mathrm{T}$ with $X \stackrel{a}{\longrightarrow} \alpha$ and all transitions $Z \stackrel{a}{\longrightarrow} Y \alpha \in \mathrm{T}$ with $Z \stackrel{a}{\longrightarrow} X \alpha$. It is clear that this transformation of a BP definition preserves weak bisimulation equivalence. We therefore assume that atoms of a BP definition adhere to the following condition: (1) if $X \neq Y$ and $X \stackrel{\epsilon}{\Longrightarrow}{ }_{0} Y$ then $\operatorname{not}\left(Y \stackrel{\epsilon}{\Longrightarrow}{ }_{0} X\right)$.

The reason that the transition relation $\stackrel{\epsilon}{\Longrightarrow} 0$ can be infinite branching is because atoms can "generate" other atoms. If $X \stackrel{\epsilon}{\Longrightarrow} X A$ then we say that $X$ generates $A$. And for each atom $X$, the set of atoms generated by $X$, written 
$G(X)$, is $\{A: X \stackrel{\epsilon}{\Longrightarrow} X A\}$. In Example 2 of the previous section, $G(D)=\emptyset$ and $G(U)=G(V)=\{D\}$.

\section{Proposition 3}

1. If $A \in G(X)$ then $\mathrm{N}(A)=0$

2. If $A \in G(X)$ and $A \stackrel{\epsilon}{\Longrightarrow} B$ then $B \in G(X)$

3. If $A \in G(B)$ and $B \in G(X)$ then $A \in G(X)$

4. If $\alpha \in G(X)^{*}$ then $X \stackrel{\epsilon}{\Longrightarrow} X \alpha$

5. If $\alpha \in G(X)^{*}$ then $X \approx X \alpha$

If $A \in G(X)$ then $A^{n+1} X \alpha \approx X \alpha$. Hence any configuration $\alpha$ can be reduced to an equivalent minimal normal form $\operatorname{nf}(\alpha)$.

Definition 2 If $\alpha=X_{1}^{k_{1}} \ldots X_{n}^{k_{n}}$ then $\operatorname{nf}(\alpha)=X_{1}^{l_{1}} \ldots X_{n}^{l_{n}}$ where

1. if $j \neq i$ and $X_{j} \in G\left(X_{i}\right)$ and $k_{i}>0$ then $l_{j}=0$,

2. if $X_{i} \in G\left(X_{i}\right)$ and $k_{i}>0$ and $\forall j \neq i$. $k_{j}=0$ or $X_{i} \notin G\left(X_{j}\right)$ then $l_{i}=1$.

3. if $k_{j}=0$ or for all $i$ such that $k_{i} \neq 0, X_{j} \notin G\left(X_{i}\right)$ then $l_{j}=k_{j}$.

\section{Proposition 4}

1. If $X_{1}^{l_{1}} \ldots X_{n}^{l_{n}}=\operatorname{nf}(\alpha)$ and $X_{1}^{j_{1}} \ldots X_{n}^{j_{n}}=\operatorname{nf}(\alpha)$ then $l_{i}=j_{i}$ for each $i$

2. $\alpha \approx \operatorname{nf}(\alpha)$

Assume a BP process definition which obeys condition (1) and let $\Gamma^{0}$ be the set of generable atoms, $\{A \in \Gamma: \exists X . A \in G(X)\}$. An "extended" configuration either has the form $\beta$ where $\beta=\operatorname{nf}(\beta)$, or has the form $\beta A_{1}^{*} \ldots A_{k}^{*}$ where $\beta=\operatorname{nf}(\beta)$ and each $A_{i} \in \Gamma^{0}$ and $A_{i} \notin G(X)$ for any $X \in \beta$, and $A_{i} \notin \beta$.

Theorem 1 For any configuration $\alpha$ and $a \in(\mathrm{A} \cup\{\epsilon\})$ and $n$ there is a finite set of extended configurations $\mathrm{E}(\alpha, a, n)$ such that

1. if $\alpha \stackrel{a}{\Longrightarrow}{ }_{n} \delta$ then either $\operatorname{nf}(\delta) \in \mathrm{E}(\alpha, a, n)$ or $\delta=\delta_{1} A_{1}^{l_{1}} \ldots A_{k}^{l_{k}}$ and each $l_{i} \geq 0$ and $\beta=\operatorname{nf}\left(\delta_{1}\right)$ and $\beta A_{1}^{*} \ldots A_{k}^{*} \in \mathrm{E}(\alpha, a, n)$,

2. if $\beta \in \mathrm{E}(\alpha, a, n)$ then $\alpha \stackrel{a}{\Longrightarrow}{ }_{n} \beta$,

3. if $\beta A_{1}^{*} \ldots A_{k}^{*} \in \mathrm{E}(\alpha, a, n)$ then $\forall l_{1} \geq 0 . \ldots \forall l_{k} \geq 0 . \quad \alpha \stackrel{a}{\Longrightarrow}{ }_{n} \beta A_{1}^{l_{1}} \ldots A_{k}^{l_{k}}$.

Proof: Assume configuration $\alpha$ and assume $a \in \mathrm{A} \cup\{\epsilon\}$ and $n \geq-1$. Any transition $\alpha \stackrel{a}{\Longrightarrow}{ }_{n} \delta$ can be decomposed as follows $\alpha \stackrel{\epsilon}{\Longrightarrow} \alpha_{1} \stackrel{a}{\longrightarrow} \delta^{\prime} \stackrel{\epsilon}{\Longrightarrow} \delta$ where $j+k+l=n$. Clearly for the set $\{\delta: \alpha \stackrel{a}{\Longrightarrow} \delta\}$ there are only finitely many different indices $j, k$ and $l$ which can be involved in a decomposition (because $k \leq \mathrm{K}$ and both $j$ and $l$ are at least 0 ). In turn a transition $\lambda \stackrel{\epsilon}{\Longrightarrow} \lambda^{\prime}$ can also be decomposed. If $m=0$ then $\lambda \stackrel{\tau}{\longrightarrow} 0 \ldots \stackrel{\tau}{\longrightarrow} \lambda_{0} \lambda^{\prime}$ and if $m>0$ then $\lambda \stackrel{\epsilon}{\Longrightarrow} \lambda_{1} \stackrel{\tau}{\longrightarrow} \lambda_{1}^{\prime} \stackrel{\epsilon}{\Longrightarrow}{ }_{m-k} \lambda^{\prime}$ where $k>0$. Hence any transition $\stackrel{a}{\Longrightarrow}{ }_{n}$ is built from only finitely many compositions of transitions $\stackrel{\epsilon}{=}, \stackrel{a}{\longrightarrow}{ }_{k}$ and $\stackrel{\tau}{\longrightarrow}$ where $m>0$. And for each $\lambda$ the sets $\left\{\lambda^{\prime}: \lambda \stackrel{a}{\longrightarrow} \lambda_{k}\right\}$ and $\left\{\lambda^{\prime}: \lambda \stackrel{\tau}{\longrightarrow}_{m} \lambda^{\prime}\right\}$ are finite and bounded, from the BP process definition. Hence the important transitions 
involved in a decomposition are the $\stackrel{\epsilon}{=}{ }_{0}$ transitions, which we now concentrate on. A transition of the form $X \stackrel{\tau}{\longrightarrow} 0$ X $X$ is a generating transition, and a transition $X \stackrel{\tau}{\longrightarrow} X$ is useless. Consider any configuration $\beta_{0}$ and derivation $d=\beta_{0} \stackrel{\tau}{\longrightarrow} 0 \beta_{1} \stackrel{\tau}{\longrightarrow} 0 \ldots \stackrel{\tau}{\longrightarrow} 0 \beta_{n}$ such that no transition in the derivation is either a generating transition or a useless transition. For a fixed $\beta_{0}$ there are only finitely many such derivations, and therefore only finitely many configurations appearing in any such derivation, $\left\{\beta_{0}, \ldots, \beta_{m}\right\}$. This follows from condition (1) earlier: if $X \stackrel{\tau^{+}}{\longrightarrow} Y \alpha$ and $Y \stackrel{\tau^{+}}{\longrightarrow} 0 X \delta$ and $X \neq Y$ then $Y$ is redundant because of $X($ as $\mathrm{N}(\alpha)=0=\mathrm{N}(\delta))$. In fact a crude upper bound on the number of such final configuration 11 is $\left|\beta_{0}\right| \times 2^{|\Gamma|}$. For each derivation $d$ of $\beta_{i}$ let $d\left(\beta_{i}\right) \subseteq \Gamma$ be the subset of atoms which occur anywhere within the derivation, and let $G\left(d\left(\beta_{i}\right)\right)$ be the set $\bigcup\left\{G(X): X \in d\left(\beta_{i}\right)\right\}$. There are only finitely many different such sets associated with each $\beta_{i}$. For each such subset we introduce a preliminary extended configuration as follows. First if $G\left(d\left(\beta_{i}\right)\right)=\emptyset$ for some derivation $d$ then one preliminary configuration is $\beta_{i}$. Next if $G\left(d\left(\beta_{i}\right)\right)=\left\{A_{1}, \ldots, A_{k}\right\}$ then another preliminary configuration is $\beta_{i}^{\prime} A_{1}^{*} \ldots A_{k}^{*}$ where $\beta_{i}^{\prime}$ is the result of removing all occurrences of $A_{j}$ from $\beta_{i}$. There are only finitely many such preliminary extended configurations associated with each $\beta_{i}$. Preliminary extended configurations are preliminary because they may not yet be in normal form. However it is easy to see that if $\beta_{0} \stackrel{\epsilon}{\Longrightarrow} \delta$ then either $\delta=\beta_{i}$ or $\delta=\beta_{i}^{\prime} A_{1}^{l_{1}} \ldots A_{k}^{l_{k}}$ for some $l_{1} \geq 0, \ldots, l_{k} \geq 0$. Moreover if $\beta_{i}^{\prime} A_{1}^{*} \ldots A_{k}^{*}$ is a preliminary extended configuration then by Proposition 3.4 for all $l_{1} \geq 0, \ldots, l_{k} \geq 0, \beta_{0} \stackrel{\epsilon}{=} \beta_{i}^{\prime} A_{1}^{l_{1}} \ldots A_{k}^{l_{k}}$. We now complete the argument of the theorem. First if $a=\epsilon$ and $n=0$ and $\alpha=\beta_{0}$ then we merely tidy the preliminary extended configurations. If $\beta_{i}$ is such a configuration then we let $\beta_{i}^{\prime \prime}=\operatorname{nf}\left(\beta_{i}\right)$, and if $\beta_{i}^{\prime} A_{1}^{*} \ldots A_{k}^{*}$ is a configuration then we let $\beta_{i}^{\prime \prime}=\operatorname{nf}\left(\beta_{i}^{\prime}\right)$ and we remove each $A_{j}^{*}$ such that $A_{j} \in G(X)$ when $X \in \beta_{i}^{\prime \prime}$. By the reasoning above the resulting finite set of extended configurations, $\mathrm{E}(\alpha, \epsilon, 0)$, obey the theorem. Otherwise $a \neq \epsilon$ or $n \neq 0$. Assume the finite set of preliminary extended forms associated with $\left\{\alpha_{1}: \alpha \stackrel{\epsilon}{\Longrightarrow} \alpha_{1}\right\}$. Consider the possible transitions $\stackrel{a}{\longrightarrow}$ from a preliminary extended form where $a \neq \tau$ or $k \neq 0$. There are two cases. First if the form is $\beta_{i}$ then the required finite set is $\left\{\beta_{i j}: \beta_{i} \stackrel{a}{\longrightarrow}_{k} \beta_{i j}\right\}$. Second is that the preliminary form is $\beta_{i}^{\prime} A_{1}^{*} \ldots A_{k}^{*}$. We now take the following finite set

$$
\left\{\beta_{i j} A_{1}^{*} \ldots A_{k}^{*}: \beta_{i}^{\prime} \stackrel{a}{\longrightarrow} \beta_{i j}\right\} \cup\left\{\beta_{i}^{\prime} \delta A_{1}^{*} \ldots A_{k}^{*}: A_{j} \stackrel{a}{\longrightarrow}_{k} \delta\right\}
$$

and then tidy their elements by removing any occurrences of $A_{j}$ from $\beta_{i j}$ and $\delta$. The result is a finite set of preliminary extended forms, with the crucial property that if $\alpha \stackrel{\epsilon}{\Longrightarrow} \alpha_{1} \stackrel{a}{\longrightarrow} \alpha_{1}^{\prime}$ then either $\alpha_{1}^{\prime}$ is a preliminary form or $\alpha_{1}^{\prime}=\beta_{i j}^{\prime} A_{1}^{l_{1}} \ldots A_{k}^{l_{k}}$ for some $l_{1} \geq 0, \ldots, l_{k} \geq 0$ and $\beta_{i j}^{\prime} A_{1}^{*} \ldots A_{k}^{*}$ is a preliminary form and for each such form and $l_{1} \geq 0, \ldots, l_{k} \geq 0$ there is an $\alpha_{1}$ such that $\alpha \stackrel{\epsilon}{\Longrightarrow} \alpha_{1} \stackrel{a}{\longrightarrow} \beta_{i j}^{\prime} A_{1}^{l_{1}} \ldots A_{k}^{l_{k}}$. The argument is now repeated, so that there is a finite set of preliminary extended forms which characterise the set $\left\{\alpha_{2}: \alpha \stackrel{\epsilon}{\Longrightarrow} 0\right.$ $\left.\alpha_{1} \stackrel{a}{\longrightarrow}{ }_{k} \alpha_{1}^{\prime} \stackrel{\epsilon}{=} 2\right\}$, and so on. Because there can be only finitely many different

\footnotetext{
${ }^{1}$ The size of a configuration $\delta,|\delta|$, is its number of occurrences of atoms.
} 
indices involved in a decomposition of the transition $\stackrel{a}{\Longrightarrow}$ it follows that there is a finite set of preliminary extended forms which characterise $\left\{\delta: \alpha \stackrel{a}{\Longrightarrow}{ }_{n} \delta\right\}$. This finite set is then tidied into a set of extended forms, $\mathrm{E}(\alpha, a, n)$, as described earlier.

Theorem 1 offers a finite symbolic characterisation of the infinite branching of normed BP processes. Moreover its proof shows how a finite set of extended configurations $\mathrm{E}(\alpha, a, n)$ which characterises $\left\{\delta: \alpha \stackrel{a}{\Longrightarrow}{ }_{n} \delta\right\}$ is computed.

Example 1 Consider $\mathrm{E}(U, c, 0)$ where $U$ is from Example 2 of the previous section. There is only one decomposition of the transition $\stackrel{c}{\Longrightarrow}{ }_{0}$ in this example, $U \stackrel{\epsilon}{\Longrightarrow} \alpha_{1} \stackrel{c}{\longrightarrow} \alpha_{1}^{\prime} \stackrel{\epsilon}{\Longrightarrow} \alpha_{0} \delta$. First consider the preliminary extended configurations for $\left\{\alpha_{1}: U \stackrel{\epsilon}{\Longrightarrow} \alpha_{1}\right\}$. This consists of the singleton set $\left\{U D^{*}\right\}$. Next we examine the $\stackrel{c}{\longrightarrow} 0$ transitions, and there are two possibilities $U \stackrel{c}{\longrightarrow}{ }_{0} U$ and $U \stackrel{c}{\longrightarrow}{ }_{0} C$. Hence $\left\{U D^{*}, C D^{*}\right\}$ contains the preliminary extended configurations for $\left\{\alpha_{1}^{\prime}: \alpha \stackrel{\epsilon}{\Longrightarrow} \alpha_{1} \stackrel{c}{\longrightarrow} \alpha_{1}^{\prime}\right\}$. This is the same set of preliminary extended configurations for $\{\delta ; \alpha \stackrel{c}{\Longrightarrow} 0 \delta$. Now we tidy this set. Because $D \in G(U)$ the resulting set $\mathrm{E}(U, c, 0)$ is $\left\{U, C D^{*}\right\}$. We show that this set obeys the three conditions of Theorem 1. Suppose $U \stackrel{c}{\Longrightarrow}{ }_{0} \delta$. There are two cases. First $U \stackrel{c}{\Longrightarrow}{ }_{0} U D^{n}$ and because $D \in G(U)$ it follows that $U=\operatorname{nf}\left(U D^{n}\right)$ and $U \in \mathrm{E}(U, c, 0)$. Second is that $U \stackrel{c}{\Longrightarrow} C D^{n}$ and $U D^{*} \in \mathrm{E}(U, c, 0)$. This establishes condition 1 of Theorem 1. For 2 note that $U \stackrel{c}{\Longrightarrow}{ }_{0} U$. And for condition $3, U \stackrel{c}{\Longrightarrow}{ }_{0} C D^{n}$ for all $n \geq 0$. In contrast the set $\mathrm{E}(V, c, 0)$, where $V$ is also from Example 2 of the previous section, is the singleton set $\left\{C D^{*}\right\}$.

\section{The Decidability Result}

Given a normed BP process definition it is easy to find its redundant atoms, and to remove them. The sets $G(X)$ for each atom $X$ is easily computable. Moreover for each configuration $\alpha, a$ and $n \leq \mathrm{K}$, one can compute a finite set of extended configurations $\mathrm{E}(\alpha, a, n)$ which characterises $\left\{\delta: \alpha \stackrel{a}{\Longrightarrow}{ }_{n} \delta\right\}$, using Theorem 1 of the previous section. The main problem with deciding whether or not $\alpha \approx \beta$ is their infinite branching. The technique for overcoming this is to use the finite characterisation to show that we only need to examine boundedly many transitions of $\alpha$ and $\beta$. However we are only able to show this for a subset of normed BP processes which includes Examples 1 and 2 of section 2 .

We restrict to the subset of normed BP process definitions which obey the following condition.

$$
\text { If } G(X) \neq \emptyset \text { and } X \stackrel{\tau}{\longrightarrow} 0 \text { then } \alpha=X \alpha^{\prime}
$$

Effectively this imposes the constraint that generators are "pure", if $X$ is a generator then any transition $X \stackrel{\tau}{\longrightarrow}{ }_{0} \alpha$ is a generating transition or is useless $(X \stackrel{\tau}{\longrightarrow} 0$ ). Both Examples 1 and 2 of section 2 obey this condition. The next result relies on this constraint. 


\section{Proposition 1}

1. If $G(X) \neq \emptyset$ and $X \alpha \stackrel{\epsilon}{\Longrightarrow}{ }_{0} \beta$ then $X \in \beta$.

2. For each $\alpha$ the set $\{\operatorname{nf}(\delta): \alpha \stackrel{\epsilon}{\Longrightarrow} \delta\}$ is finite.

The restriction on BP processes does not imply that the sets $\{\delta: \alpha \stackrel{\epsilon}{\Longrightarrow} \delta\}$ and $\left\{\operatorname{nf}(\delta): \alpha \stackrel{a}{\Longrightarrow}{ }_{n} \delta\right.$ and $a \neq \epsilon$ or $\left.n>0\right\}$ are finite 2 .

Assume that $\mathrm{E}(\alpha, a, n)$ is the finite set of extended configurations given by Theorem 1 of the previous section, which characterises $\left\{\delta: \alpha \stackrel{a}{{ }_{n}} \delta\right\}$. Elements of this set are either finite, of the form $\beta$, or infinite, of the form $\beta A_{1}^{*} \ldots A_{k}^{*}$. It follows from the restriction on $\mathrm{BP}$ processes that if $\beta$ is a finite element and $\beta \stackrel{\epsilon}{\Longrightarrow} \beta^{\prime}$ then $\mathrm{nf}\left(\beta^{\prime}\right)$ is also a finite element because if $\beta$ contains a generator $X$ then $\beta^{\prime}$ also contains $X$. Furthermore if $\beta \stackrel{\epsilon}{\Longrightarrow} \beta^{\prime}$ and $\operatorname{nf}\left(\beta^{\prime}\right) \neq \beta$ and $\beta^{\prime} \stackrel{\epsilon}{\Longrightarrow} \beta^{\prime \prime}$ then $\operatorname{nf}\left(\beta^{\prime \prime}\right) \neq \beta$ (because otherwise $\beta^{\prime}$ would contain a redundant atom). Therefore the following holds because of the restriction on processes.

Fact 1 If $\beta_{0}$ is a finite element of $\mathrm{E}(\alpha, a, n)$ whose size $|\mathrm{E}(\alpha, a, n)|=m$ and $\beta_{0} \stackrel{\epsilon}{\Longrightarrow} \beta_{1} \stackrel{\epsilon}{\Longrightarrow} \ldots \stackrel{\epsilon}{\Longrightarrow} \beta_{m}$ then for some $i<m, \operatorname{nf}\left(\beta_{i}\right)=\operatorname{nf}\left(\beta_{i+1}\right)$.

There is a similar property in the case of infinite elements. Assume that $\delta=$ $\beta A_{1}^{n_{1}} \ldots A_{k}^{n_{k}}$ is an instance of an infinite element $\beta A_{1}^{*} \ldots A_{k}^{*} \in \mathrm{E}(\alpha, a, n)$ and $\delta \stackrel{\epsilon}{\Longrightarrow} \delta_{0}$. Then $\delta^{\prime} \approx \lambda A_{1}^{l_{1}} \ldots A_{k}^{l_{k}}$ where each $l_{i} \leq n_{i}$ and either $\operatorname{nf}(\lambda)=\beta$ or $\operatorname{nf}(\lambda) \neq \beta$ and $\beta \stackrel{\epsilon}{\Longrightarrow}{ }_{0} \lambda$. Moreover in the case that $\operatorname{nf}(\lambda) \neq \beta$ and $\delta^{\prime} \stackrel{\epsilon}{\Longrightarrow} 0$ $\lambda^{\prime} A_{1}^{l_{1}^{\prime}} \ldots A_{k}^{l_{k}^{\prime}}$ then $\operatorname{nf}\left(\lambda^{\prime}\right) \neq \beta$.

Fact 2 If $\delta_{0}=\beta A_{1}^{n_{1}} \ldots A_{k}^{n_{k}}$ and $\beta A_{1}^{*} \ldots A_{k}^{*} \in \mathrm{E}(\alpha, a, n)$ and $|\mathrm{E}(\alpha, a, n)|=m$ and $\delta_{0} \stackrel{\epsilon}{\Longrightarrow} \delta_{1} \stackrel{\epsilon}{\Longrightarrow} \ldots \stackrel{\epsilon}{\Longrightarrow}{ }_{0} \delta_{m}$ then for some $i<m, \delta_{i} \approx \lambda_{i} A_{1}^{l_{1}} \ldots A_{k}^{l_{k}}$ and $\delta_{i+1} \approx \lambda_{i}^{\prime} A_{1}^{l_{1}^{\prime}} \ldots A_{k}^{l_{k}^{\prime}}$ and $\operatorname{nf}\left(\lambda_{i}\right)=\operatorname{nf}\left(\lambda_{i}^{\prime}\right)$ and $l_{i}^{\prime} \leq l_{i}$.

Stratified weak bisimulation approximants, $\approx_{o}^{\prime}$, are now defined as follows.

Definition 1 The relations $\approx_{o}^{\prime}$ for ordinals $o$ are defined inductively as follows, where we assume that $l$ is a limit ordinal (such as $\omega$ ).

$$
\begin{aligned}
& \alpha \approx_{0}^{\prime} \beta \\
& \alpha \approx_{o+1}^{\prime} \beta \text { iff } \\
& \text { if } \alpha \stackrel{\epsilon}{\Longrightarrow} \alpha_{0} \text { then } \exists \beta^{\prime} . \beta \stackrel{\epsilon}{\Longrightarrow} \beta_{0} \text { and } \alpha^{\prime} \approx_{o+1}^{\prime} \beta^{\prime} \\
& \text { if } \beta \stackrel{\epsilon}{\Longrightarrow} \beta_{0} \text { then } \exists \alpha^{\prime} . \alpha \stackrel{\epsilon}{\Longrightarrow} \alpha_{0} \alpha^{\prime} \text { and } \alpha^{\prime} \approx_{o+1}^{\prime} \beta^{\prime} \\
& \text { and for }(a \in \mathrm{A} \text { and } n \leq K) \text { or }(a=\epsilon \text { and } 1 \leq n \leq \mathrm{K}) \\
& \text { if } \alpha \stackrel{a}{\Longrightarrow}{ }_{n} \alpha^{\prime} \text { then } \exists \beta^{\prime} . \beta \stackrel{a}{\Longrightarrow} \beta_{n} \text { and } \alpha^{\prime} \approx_{o}^{\prime} \beta^{\prime} \\
& \text { if } \beta \stackrel{a}{\Longrightarrow} \beta_{n} \text { then } \exists \alpha^{\prime} . \alpha \stackrel{a}{\Longrightarrow}{ }_{n} \alpha^{\prime} \text { and } \alpha^{\prime} \approx_{o}^{\prime} \beta^{\prime} \\
& \alpha \approx_{l}^{\prime} \beta \text { iff } \forall o<l . \alpha \approx_{o}^{\prime} \beta
\end{aligned}
$$

\footnotetext{
${ }^{2}$ It is possible to define hierarchies of BP processes according to finiteness of these sets. At the lowest level are the totally normed processes, where for any $\alpha, a$ and $n$ the set $\{\delta: \alpha \stackrel{a}{\Longrightarrow} n \delta$ is finite.
} 
The definition of $\approx_{o}^{\prime}$ is unusual because we distinguish between $\stackrel{\epsilon}{=}$ transitions and the remaining transitions $\stackrel{a}{\Longrightarrow}$ for reasons that will become clearer in the decision procedure. Because of Proposition 1 the definition is well-defined, as there can only be finitely many "different" elements in the set $\{\delta: \alpha \stackrel{\epsilon}{\Longrightarrow} \delta \delta$. For each ordinal $o$, the relation $\approx_{o}^{\prime}$ is an equivalence relation. However for particular ordinals $o$ the relation $\approx_{o}^{\prime}$ may differ from $\approx_{o}$ as defined in section 2 .

\section{Proposition 2}

1. $\alpha \approx \beta$ iff for all ordinals o. $\alpha \approx_{o}^{\prime} \beta$.

2. For all ordinals o, $\alpha \approx_{o}^{\prime} \operatorname{nf}(\alpha)$.

3. If $\alpha \approx_{o}^{\prime} \beta$ then $\alpha \delta \approx_{o}^{\prime} \beta \delta$.

The procedure for deciding whether $\alpha \approx \beta$ is given by a tableau proof system, which is goal directed. One starts with the initial goal $\operatorname{nf}(\alpha)=\operatorname{nf}(\beta)$, to be understood as "is $\alpha \approx \beta$ ?", and then one reduces it to subgoals using a small number of rules. Goal reduction continues until one reaches either obviously true goals (such as $\delta=\delta$ ) or obviously false subgoals (such as $\gamma=\delta$ and one of these processes has an $\stackrel{a}{\Longrightarrow}$ transition which the other does not have). Such a procedure was used for deciding strong bisimilarity between arbitrary BP processes [4, and we will make use of this decidability proof.

Goals have the form $\alpha=\gamma$ where $\alpha=\operatorname{nf}(\alpha)$ and $\gamma=\operatorname{nf}(\gamma)$. There are four reduction rules, reducing goals to subgoals. Let $a_{n}(\delta)=\left\{\operatorname{nf}\left(\delta^{\prime}\right): \delta \stackrel{a}{\Longrightarrow}{ }_{n} \delta^{\prime}\right\}$ where $n \leq \mathrm{K}$. The first tableau rule is for $\stackrel{\epsilon}{\Longrightarrow}$ transitions, and by Proposition 1.2 the set $\epsilon_{0}(\delta)$ is a finite set.

$$
\frac{2=\gamma}{\alpha_{1}=\gamma_{1} \quad \cdots \quad \alpha_{l}=\gamma_{l}} \mathrm{C}
$$

where $\mathrm{C}$ is the following condition

$$
\begin{aligned}
& \left(\forall \alpha^{\prime} \in \epsilon_{0}(\alpha) . \exists i . \alpha^{\prime}=\alpha_{i}\right) \wedge\left(\forall i . \alpha_{i} \in \epsilon_{0}(\alpha)\right) \wedge \\
& \left(\forall \gamma^{\prime} \in \epsilon_{0}(\gamma) . \exists i . \gamma^{\prime}=\gamma_{i}\right) \wedge\left(\forall i . \gamma_{i} \in \epsilon_{0}(\gamma)\right)
\end{aligned}
$$

The rule is sound, if all the subgoals are true then so is the goal. A finer account shows soundness with respect to approximants, if $\alpha_{i} \approx_{o}^{\prime} \gamma_{i}$ for all subgoals then $\alpha \approx_{o}^{\prime} \gamma$. The rule is also complete in the sense that if the goal $\alpha=\gamma$ is true then there is an application of the rule such that all subgoals $\alpha_{i}=\gamma_{i}$ are also true.

Next we want a similar rule which covers the remaining transitions $\stackrel{a}{\Longrightarrow}$, when $n \leq \mathrm{K}$ and either $a \neq \epsilon$ or $n>0$. A similar rule would reduce a goal $\alpha=\gamma$ to only a finite set of subgoals. However a set $a_{n}(\delta)$ may be infinite. Therefore we need a mechanism which shows that we only need to consider bounded finite subsets of elements of $a_{n}(\alpha)$ and $a_{n}(\gamma)$ for each $\stackrel{a}{\Longrightarrow}$. Lemmas 1 and 2 below establish this. These results are quite involved, and so we delay their proofs until the next section where they are presented in full. They constitute the heart of the decidability result.

Although the set $a_{n}(\delta)$ may be infinite, the set $\mathrm{E}(\delta, a, n)$ is not only finite but also bounded. We show that we need only examine "small" elements of $a_{n}(\delta)$. 
The first lemma covers the case when the goal $\alpha=\gamma$ is not true. Without loss of generality assume $\alpha \not_{o+1}^{\prime} \gamma$ and $\alpha \stackrel{a}{\Longrightarrow}{ }_{n} \alpha^{\prime}$ and for all $\gamma^{\prime}$ such that $\gamma \stackrel{a}{\Longrightarrow}{ }_{n} \gamma^{\prime}$, $\alpha^{\prime} \not_{o}^{\prime} \gamma^{\prime}$. Lemma 1, the bounded inequivalence lemma, shows that there is a small $\alpha^{\prime} \in a_{n}(\alpha)$ with this property. A small element of $a_{n}(\alpha)$ is either a finite element of $\mathrm{E}(\alpha, a, n)$ or is an element $\beta A_{1}^{l_{1}} \ldots A_{k}^{l_{k}}$ where each $l_{i} \leq|\mathrm{E}(\gamma, a, n)|+1$ and $\beta A_{1}^{*} \ldots A_{k}^{*} \in \mathrm{E}(\alpha, a, n)$.

Lemma 1 Let $\alpha \not_{o+1}^{\prime} \gamma$ and assume that $\alpha \stackrel{a}{\Longrightarrow}{ }_{n} \alpha^{\prime}$ and for all $\gamma^{\prime}$ such that $\gamma \stackrel{a}{\Longrightarrow} \gamma^{\prime}, \alpha^{\prime} \not_{o}^{\prime} \gamma^{\prime}$. Then either

1. $\operatorname{nf}\left(\alpha^{\prime}\right)$ is a finite element in $\mathrm{E}(\alpha, a, n)$ or

2. $\alpha^{\prime}=\delta A_{1}^{n_{1}} \ldots A_{k}^{n_{k}}$ and $\beta A_{1}^{*} \ldots A_{k}^{*} \in \mathrm{E}(\alpha, a, n)$ and $\operatorname{nf}(\delta)=\beta$ and there exists $l_{1}, \ldots, l_{k}$ such that each $l_{i} \leq|\mathrm{E}(\gamma, a, n)|+1$ and for all $\gamma^{\prime}$ such that $\gamma \stackrel{a}{\Longrightarrow}{ }_{n} \gamma^{\prime}$, $\beta A_{1}^{l_{1}} \ldots A_{k}^{l_{k}} \not 火_{o}^{\prime} \gamma^{\prime}$.

Lemma 2, the bounded equivalence lemma, covers the case when $\alpha=\gamma$ is true. It shows that small elements of $a_{n}(\alpha)$ can be matched with elements of $a_{n}(\gamma)$ which have bounded size.

Lemma 2 Assume $\alpha \approx \gamma$. Then for each a and $n$

1. if $\alpha \stackrel{a}{\Longrightarrow}{ }_{n} \alpha^{\prime}$ and $\mathrm{nf}\left(\alpha^{\prime}\right) \in \mathrm{E}(\alpha, a, n)$ then

a) either $\gamma \stackrel{a}{\Longrightarrow} \gamma^{\prime}$ and $\operatorname{nf}\left(\gamma^{\prime}\right) \in \mathrm{E}(\gamma, a, n)$ and $\operatorname{nf}\left(\alpha^{\prime}\right) \approx \operatorname{nf}\left(\gamma^{\prime}\right)$

b) or $\gamma \stackrel{a}{\Longrightarrow} \lambda B_{1}^{s_{1}} \ldots B_{m}^{s_{m}}$ and $\lambda^{\prime} B_{1}^{*} \ldots B_{m}^{*} \in \mathrm{E}(\gamma, a, n)$ and $\operatorname{nf}(\lambda)=\lambda^{\prime}$ and each $s_{i} \leq|\mathrm{E}(\alpha, a, n)|$ and $\operatorname{nf}\left(\alpha^{\prime}\right) \approx \lambda^{\prime} B_{1}^{s_{1}} \ldots B_{m}^{s_{m}}$,

2. if $\alpha \stackrel{a}{\Longrightarrow}{ }_{n} \delta A_{1}^{n_{1}} \ldots A_{k}^{n_{k}}$ and $\beta A_{1}^{*} \ldots A_{k}^{*} \in \mathrm{E}(\alpha, a, n)$ and $\beta=\operatorname{nf}(\delta)$ and each $n_{i} \leq|\mathrm{E}(\gamma, a, n)|+1$ then

a) either $\gamma \stackrel{a}{\Longrightarrow} \gamma^{\prime}$ and $\operatorname{nf}\left(\gamma^{\prime}\right) \in \mathrm{E}(\gamma, a, n)$ and $\beta A_{1}^{n_{1}} \ldots A_{k}^{n_{k}} \approx \operatorname{nf}\left(\gamma^{\prime}\right)$

b) or $\gamma \stackrel{a}{\Longrightarrow} \lambda B_{1}^{s_{1}} \ldots B_{m}^{s_{m}}$ and $\lambda^{\prime} B_{1}^{*} \ldots B_{m}^{*} \in \mathrm{E}(\gamma, a, n)$ and $\mathrm{nf}(\lambda)=\lambda^{\prime}$ and each $s_{i} \leq \sum n_{i}+|\mathrm{E}(\alpha, a, n)|$ and $\beta A_{1}^{n_{1}} \ldots A_{k}^{n_{k}} \approx \lambda^{\prime} B_{1}^{s_{1}} \ldots B_{m}^{s_{m}}$.

Consequently the second tableau proof rule has a similar form to the $\stackrel{\epsilon}{\Longrightarrow} 0$ transition rule except that the condition $\mathrm{C}$ is that for all $a_{n}, n \leq \mathrm{K}$ and $a \neq \epsilon$ or $n>0$,

$\forall$ small $\alpha^{\prime} \in a_{n}(\alpha) . \exists i . \alpha=\alpha_{i} \wedge \forall i . \exists a_{n} . \alpha_{i} \in a_{n}(\alpha)$ and $\alpha_{i}$ has bounded size $\wedge$ $\forall$ small $\gamma^{\prime} \in a_{n}(\gamma) . \exists i \cdot \gamma^{\prime}=\gamma_{i} \wedge \forall i . \exists a_{n} \cdot \gamma_{i} \in a_{n}(\gamma)$ and $\gamma_{i}$ has bounded size

where the precise notion of bounded size is given in Lemma 2.2 part (b). Lemma 1 guarantees that the rule is sound, if the goal $\alpha=\gamma$ is false and so $\alpha \neq_{o+1}^{\prime} \gamma$ then for at least one of the subgoals $\alpha_{i}=\gamma_{i}$ it is the case that $\alpha_{i} \not^{\prime}{ }_{o} \gamma_{i}$ (where the approximant index decreases). Lemma 2 justifies completeness, if the goal $\alpha=\gamma$ is true then there is an application of the rule such that all subgoals $\alpha_{i}=\gamma_{i}$ are also true.

The final two rules $\mathrm{SUB}(\mathrm{L})$ and $\mathrm{SUB}(\mathrm{R})$ are taken from the tableau decision procedure for strong bisimulation equivalence for arbitrary BP processes [4]. We assume a fixed linear ordering $<$ on the atoms $\Gamma$ which is defined so that if atom $Y \in G(X)$ and $Y \neq X$ then $X<Y$. The ordering $<$ is extended to 
configurations, as the lexicographical ordering. Assume $X_{1}<X_{2}<\ldots<X_{n}$. Consequently

$$
\begin{gathered}
X_{1}^{k_{1}} \ldots X_{n}^{k_{n}}<X_{1}^{l_{1}} \ldots X_{n}^{l_{n}} \text { iff there is an } i \geq 1 \text { such that } k_{i}<l_{i} \\
\text { and for all } j<i . k_{j}=l_{j} .
\end{gathered}
$$

Clearly, $\operatorname{nf}(\alpha) \leq \alpha$ and if $\alpha<\gamma$ then $\operatorname{nf}(\alpha \beta)<\operatorname{nf}(\gamma \beta)$.

The SUB rules are given below, where $\mathrm{SUB}(\mathrm{L})$ is the left rule and $\mathrm{SUB}(\mathrm{R})$ is the right rule.

$$
\begin{array}{ccccc}
\alpha=\gamma & & \gamma=\alpha & \\
\vdots & \gamma<\alpha \text { and at least one } & \vdots & \gamma<\alpha \text { and at least one } \\
\vdots & \text { application of } \stackrel{a}{\Longrightarrow} & \vdots & \text { application of }{ }_{n} \\
\frac{\alpha \delta=\lambda}{\operatorname{nf}(\gamma \delta)=\lambda} & & \frac{\lambda=\alpha \delta}{\lambda=\operatorname{nf}(\gamma \delta)}
\end{array}
$$

We explain the rule $\mathrm{SUB}(\mathrm{L})$. If the current goal is $\alpha \delta=\lambda$ and in the proof tree above the goal on the path to the root there is the goal $\alpha=\gamma$ and $\gamma<\alpha$ and there is at least one application of the rule $\stackrel{a}{\Longrightarrow}$ where $a \neq \epsilon$ or $n>0$ along this path then we reduce the goal to the subgoal $\operatorname{nf}(\gamma \delta)=\lambda$. Note that this has the effect of reducing the size of the left configuration, as $\operatorname{nf}(\gamma \delta)<\alpha \delta$. The SUB rules are sound and complete.

\section{Fact 3}

1. If $\alpha \delta \not_{o}^{\prime} \lambda$ and $\alpha \approx_{o}^{\prime} \gamma$ then $\operatorname{nf}(\gamma \delta) \not \neq_{o}^{\prime} \lambda$

2. If $\alpha \approx \gamma$ and $\alpha \delta \approx \lambda$ then $\operatorname{nf}(\gamma \delta) \approx \lambda$

One builds a proof tree starting from an initial goal $\alpha=\gamma$ and repeatedly applying the tableau proof rules as follows. First one applies the $\stackrel{\epsilon}{\Longrightarrow} 0$ rule and then one applies the $\stackrel{a}{\Longrightarrow}$ rule to all the resulting subgoals. Call this a simple block. And then one repeatedly applies the SUB rules to the subgoals of a block, until they no longer apply. At which point the whole process is repeated. One applies the $\stackrel{\epsilon}{=} 0$ rule to all subgoals and so on.

There is also the important notion of when a goal is a final goal. Final goals are either successful or unsuccessful. A successful final goal has the form $\alpha=\alpha$ and an unsuccessful final goal has the form $\delta=\lambda$ and for some $a$ and $n$ either $a_{n}(\delta)=\emptyset$ and $a_{n}(\lambda) \neq \emptyset$ or $a_{n}(\delta) \neq \emptyset$ and $a_{n}(\lambda)=\emptyset$. Clearly a successful final goal is true and an unsuccessful final goal is false.

A successful tableau proof for $\alpha=\gamma$ is a finite proof tree whose root is $\alpha=\gamma$ and all of whose inner subgoals are the result of an application of one of the rules, and all of whose leaves are successful final goals. The following results establish decidability of $\approx$ between restricted BP processes. The proofs of these results are minor variants of proofs in 34 .

Theorem 1 Every tableau for $\alpha=\gamma$ is finite and there is only a finite number of tableau for $\alpha=\gamma$. 
Theorem $2 \alpha \approx \gamma$ iff there is a successful tableau for $\alpha=\gamma$.

Therefore the main result follows that $\approx$ is decidable between restricted BP processes. We now examine how this result shows equivalence and inequivalence for the examples from section 2 .

Example 1 We show that $Y \approx Z$ where these atoms are from Example 1 of section 2. Assume $Y<Z$. We build a tableau with root $Y=Z$. First we apply the $\stackrel{\epsilon}{\Longrightarrow}$ rule which results in the same goal $Y=Z$. Then we apply the $\stackrel{a}{\Longrightarrow}_{n}$ rule. In this example $K=1$ and so we need to consider the four transitions $\stackrel{\epsilon}{\Longrightarrow}$, $\stackrel{a}{\Longrightarrow}{ }_{-1}, \stackrel{a}{\Longrightarrow} 0$ and $\stackrel{a}{\Longrightarrow}$.

$$
\begin{aligned}
& \epsilon_{0}(Y)=\{Y A\} \quad a_{-1}(Y)=\{A\} \quad a_{0}(Y)=\{A, Y\} \quad a_{1}(Y)=\{Y A, A A\} \\
& \epsilon_{0}(Z)=\{Z A\} \quad a_{-1}(Z)=\{A\} \quad a_{0}(Z)=\{A, Z\} \quad a_{1}(Z)=\{Z A, A A\}
\end{aligned}
$$

So the goal $Y=Z$ reduces to the following subgoals, (1) $Y A=Z A,(2) A=A$, (3) $Y=Z$ and (4) $A A=A A$. Goals (2) and (4) are successful leaves. In the cases of (1) and (3) because $Y<Z$ and $Y=Z$ appears on their paths to the root, and in both cases there is at least one application of the rule $\stackrel{a}{\Longrightarrow}_{n}$, we can apply $\mathrm{SUB}(\mathrm{R})$ to yield $\left(1^{\prime}\right) Y A=Y A$ and $\left(3^{\prime}\right) Y=Y$, which completes the successful tableau.

Example 2 We show that $U \not V$ where $U, V$ are from Example 2 of section 2 even though $U \approx_{n}^{\prime} V$ for all $n \geq 0$. There are only finitely many tableaux for this goal and all are unsuccessful. Assume that $U<V$. The starting goal is $U=V$. First we apply the $\stackrel{\epsilon}{\Longrightarrow}$ rule, which yields the same goal $U=V$. For this example $\mathrm{K}=0$. Next we apply the $\stackrel{a}{\Longrightarrow}$ rule and there are two possible transitions $\stackrel{c}{\Longrightarrow} 0$ and $\stackrel{d}{\Longrightarrow}$. We only examine the first of these. $\mathrm{E}(U, c, 0)=\left\{U, C D^{*}\right\}$ and $\mathrm{E}(V, c, 0)=\left\{C D^{*}\right\}$. The small elements of $c_{0}(U)=\left\{U, C, C D, C D^{2}\right\}$ and the small elements of $c_{0}(V)=\left\{C, C D, C D^{2}, C D^{3}\right\}$. Therefore we must find a matching of small elements with bounded elements. The easy subgoals are $C=C, C D=C D, C D^{2}=C D^{2}$ and $C D^{3}=C D^{3}$. The problem case is a match for the small element $U \in c_{0}(U)$. By Lemma 2 because $U$ is a finite element of $\mathrm{E}(U, c, 0)$ the matching element must be $C D^{s}$ where $s \leq 2$. Thus we must have one of the following subgoals $(1) U=C,(2) U=C D,(3) U=C D^{2}$. Assume it is (3). We apply the $\stackrel{\epsilon}{\Longrightarrow}$ rule to this goal, $\epsilon_{0}\left(C D^{2}\right)=\left\{C, C D, C D^{2}\right\}$ and $\epsilon_{0}(U)=\{U\}$. So in fact we must have all the subgoals (1), (2) and (3). But now we have an unsuccessful leaf $(1)$, because $d_{0}(U)=\{U\}$ and $d_{0}(C)=\emptyset$.

\section{Proofs of the Main Lemmas}

In this section we prove the two boundedness lemmas of the previous section.

Proof of Lemma 1: Assume $\alpha \stackrel{a}{\Longrightarrow}{ }_{n} \alpha^{\prime}$ and $\alpha^{\prime} \not_{o}^{\prime} \gamma^{\prime}$ for all $\gamma^{\prime}$ such that $\gamma \stackrel{a}{\Longrightarrow} \gamma^{\prime}$. Consider the sets $\mathrm{E}(\alpha, a, n)$ and $\mathrm{E}(\gamma, a, n)$. By Theorem 1 of section 3 either $\operatorname{nf}\left(\alpha^{\prime}\right) \in \mathrm{E}(\alpha, a, n)$ or $\alpha^{\prime}=\delta A_{1}^{n_{1}} \ldots A_{k}^{n_{k}}$ and $\beta A_{1}^{*} \ldots A_{k}^{*} \in \mathrm{E}(\alpha, a, n)$ and $\operatorname{nf}(\delta)=\beta$. If the first holds then the result is proved. Assume therefore that 
$\beta A_{1}^{n_{1}} \ldots A_{k}^{n_{k}} \not_{o}^{\prime} \gamma^{\prime}$ for all $\gamma^{\prime}$ such that $\gamma \stackrel{a}{\Longrightarrow}{ }_{n} \gamma^{\prime}$. Let $\beta_{1}^{\prime}=\beta A_{2}^{n_{2}} \ldots A_{k}^{n_{k}}$, and consider the least $m_{1}$ such that $\beta_{1}^{\prime} A_{1}^{m_{1}} \not_{o}^{\prime} \gamma^{\prime}$ for all $\gamma^{\prime}$ such that $\gamma \stackrel{a}{\Longrightarrow}{ }_{n} \gamma^{\prime}$. If $m_{1} \leq|\mathrm{E}(\gamma, a, n)|+1$ then the result is proved for $l_{1}=m_{1}$. The argument is then repeated for other $A_{i}$. Let $\beta_{2}^{\prime}=\beta A_{1}^{m_{1}} A_{3}^{n_{3}} \ldots A_{k}^{n_{k}}$ and consider the least $m_{2}$ such that $\beta_{2}^{\prime} A_{2}^{m_{2}} \not_{o}^{\prime} \gamma^{\prime}$ for all $\gamma^{\prime}$ such that $\gamma \stackrel{a}{\Longrightarrow_{n}} \gamma^{\prime}$. Therefore without loss of generality assume that $\beta_{1}^{\prime} A_{1}^{m_{1}} \not_{o}^{\prime} \gamma^{\prime}$ for all $\gamma^{\prime}$ such that $\gamma \stackrel{a}{\Longrightarrow}{ }_{n} \gamma^{\prime}$ and $m_{1}>|\mathrm{E}(\gamma, a, n)|+1$. Hence there is a $\gamma^{\prime}$ such that $\gamma \stackrel{a}{\Longrightarrow}{ }_{n} \gamma^{\prime}$ and $\beta_{1}^{\prime} A_{1}^{m_{1}-1} \approx_{o}^{\prime} \gamma^{\prime}$. By Theorem 1 of section 3 either $\operatorname{nf}\left(\gamma^{\prime}\right) \in \mathrm{E}(\gamma, a, n)$ or $\gamma^{\prime}=\lambda B_{1}^{s_{1}} \ldots B_{m}^{s_{m}}$ and $\lambda^{\prime} B_{1}^{*} \ldots B_{m}^{*} \in \mathrm{E}(\gamma, a, n)$ and $\operatorname{nf}(\lambda)=\lambda^{\prime}$. Assume the first case, that $\operatorname{nf}\left(\gamma^{\prime}\right)=\gamma_{0}^{\prime \prime} \in \mathrm{E}(\gamma, a, n)$. Hence $\beta_{1}^{\prime} A_{1}^{m_{1}-1} \approx_{o}^{\prime} \gamma_{0}^{\prime \prime}$. However $\beta_{1}^{\prime} A_{1}^{m_{1}-1} \stackrel{\epsilon}{\Longrightarrow} \beta_{1}^{\prime} A_{1}^{m_{1}-2} \stackrel{\epsilon}{\Longrightarrow}{ }_{0} \ldots \stackrel{\epsilon}{\Longrightarrow}{ }_{0} \beta_{1}^{\prime} A_{1}^{0}$. So therefore $\gamma_{0}^{\prime \prime} \stackrel{\epsilon}{\Longrightarrow} \gamma_{1}^{\prime \prime} \stackrel{\epsilon}{\Longrightarrow} 0$ $\ldots \stackrel{\epsilon}{\Longrightarrow} \gamma_{m_{1}-1}^{\prime \prime}$ and $\gamma_{j}^{\prime \prime} \approx_{o}^{\prime} \beta_{1}^{\prime} A_{1}^{m_{1}-(j+1)}$. By Fact 1 of the previous section there is an $i, \operatorname{nf}\left(\gamma_{i}^{\prime \prime}\right)=\operatorname{nf}\left(\gamma_{i+1}^{\prime \prime}\right)$ and therefore $\beta_{1}^{\prime} A_{1}^{m_{1}-(i+2)} \approx_{o}^{\prime} \beta_{1}^{\prime} A_{1}^{m_{1}-(i+1)}$. Because $\approx_{o}^{\prime}$ is a congruence it follows that $\beta_{1}^{\prime} A_{1}^{m_{1}-(i+2)} \approx_{o}^{\prime} \beta_{1}^{\prime} A_{1}^{m_{1}}$ which is a contradiction. Next we consider the other case, $\beta_{1}^{\prime} A_{1}^{m_{1}-1} \approx_{o}^{\prime} \gamma_{0}^{\prime}$ and $\gamma_{0}^{\prime}=\lambda B_{1}^{s_{1}} \ldots B_{m}^{s_{m}}$ and $\lambda^{\prime} B_{1}^{*} \ldots B_{1}^{*} \in \mathrm{E}(\gamma, a, n)$ and $\operatorname{nf}(\lambda)=\lambda^{\prime}$. The argument proceeds as above. $\beta_{1}^{\prime} A_{1}^{m_{1}-1} \stackrel{\epsilon}{\Longrightarrow} \beta_{1}^{\prime} A_{1}^{m_{1}-2} \stackrel{\epsilon}{\Longrightarrow} \quad \ldots \stackrel{\epsilon}{\Longrightarrow} \beta_{0}^{\prime} A_{1}^{0}$ Therefore $\gamma_{0}^{\prime} \stackrel{\epsilon}{\Longrightarrow} \gamma_{1}^{\prime} \stackrel{\epsilon}{\Longrightarrow}{ }_{0} \ldots \stackrel{\epsilon}{\Longrightarrow} \gamma_{m_{1}-1}^{\prime}$ and $\gamma_{j}^{\prime} \approx_{o}^{\prime} \beta_{1}^{\prime} A_{1}^{m_{1}-(j+1)}$. By Fact 2 of the previous section for some $i, \gamma_{i}^{\prime} \approx \lambda_{i} B_{1}^{t_{1}} \ldots B_{m}^{t_{m}}$ and $\gamma_{i+1}^{\prime} \approx \lambda_{i}^{\prime} B_{1}^{t_{1}^{\prime}} \ldots B_{m}^{t_{m}^{\prime}}$ and $t_{i}^{\prime} \leq t_{i}$ and $\operatorname{nf}\left(\lambda_{i}\right)=\operatorname{nf}\left(\lambda_{i}^{\prime}\right) \cdot \gamma_{i+1}^{\prime} \approx_{o}^{\prime} \beta_{1}^{\prime} A_{1}^{m_{1}-(i+2)}$ and $\gamma_{i}^{\prime} \approx_{o}^{\prime} \beta_{1}^{\prime} A_{1}^{m_{1}-(i+1)}$. Let $\eta=B_{1}^{t_{1}-t_{1}^{\prime}} \ldots B_{m}^{t_{m}-t_{m}^{\prime}}$. By congruence, $\gamma_{i+1}^{\prime} \eta \approx_{o}^{\prime} \beta_{1}^{\prime} A_{1}^{m_{1}-(i+2)} \eta \approx_{o}^{\prime} \beta_{1}^{\prime} A_{1}^{m_{1}-(i+1)}$. Therefore by congruence $\gamma_{i+1}^{\prime} \eta^{i+2} \approx_{o}^{\prime} \beta_{1}^{\prime} A_{1}^{m_{1}}$ which is a contradiction.

Proof of Lemma 2: Assume $\alpha \approx \gamma$. First also assume that $\alpha \stackrel{a}{\Longrightarrow}{ }_{n} \alpha^{\prime}$ and $\operatorname{nf}\left(\alpha^{\prime}\right) \in \mathrm{E}(\alpha, a, n)$. Hence $\gamma \stackrel{a}{\Longrightarrow}{ }_{n} \gamma^{\prime}$ and $\operatorname{nf}\left(\alpha^{\prime}\right) \approx \gamma^{\prime}$. By Theorem 1 of section 3 either $\operatorname{nf}\left(\gamma^{\prime}\right) \in \mathrm{E}(\gamma, a, n)$ and $\operatorname{nf}\left(\alpha^{\prime}\right)=\operatorname{nf}\left(\gamma^{\prime}\right)$, or $\gamma^{\prime}=\lambda B_{1}^{s_{1}} \ldots B_{m}^{s_{m}}$ and $\lambda^{\prime} B_{1}^{*} \ldots B_{m}^{*} \in \mathrm{E}(\gamma, a, n)$ and $\operatorname{nf}(\lambda)=\lambda^{\prime}$ and $\operatorname{nf}\left(\alpha^{\prime}\right)=\lambda^{\prime} B_{1}^{s_{1}} \ldots B_{m}^{s_{m}}$. We show that each $s_{i}$ can be chosen so that $s_{i} \leq|\mathrm{E}(\alpha, a, n)|$. The strategy for proving this is similar to the proof of Lemma 1 above. Let $\lambda^{\prime \prime}=\lambda^{\prime} B_{2}^{s_{2}} \ldots B_{m}^{s_{m}}$ and let $m_{1}$ be the smallest index such that $\operatorname{nf}\left(\alpha^{\prime}\right) \approx \lambda^{\prime \prime} B_{1}^{m_{1}}$. If $m_{1} \leq|\mathrm{E}(\alpha, a, n)|$ then we let $s_{1}=m_{1}$ and repeat the argument for the other indices $s_{i}$. Therefore assume that $m_{1}>|\mathrm{E}(\alpha, a, n)|$. However $\lambda^{\prime \prime} B_{1}^{m_{1}} \stackrel{\epsilon}{\Longrightarrow} \lambda_{0} \lambda^{\prime \prime} B_{1}^{m_{1}-1} \stackrel{\epsilon}{\Longrightarrow}{ }_{0} \ldots \stackrel{\epsilon}{\Longrightarrow}{ }_{0}$ $\lambda^{\prime \prime} B_{1}^{0}$. Therefore assuming $\alpha_{0}=\operatorname{nf}\left(\alpha^{\prime}\right), \alpha_{0} \stackrel{\epsilon}{\Longrightarrow} \alpha_{0} \stackrel{\epsilon}{\Longrightarrow} \alpha_{0} \ldots \stackrel{\epsilon}{\Longrightarrow} \alpha_{0} \alpha_{m_{1}}$ and $\alpha_{j} \approx \lambda^{\prime \prime} B_{1}^{m_{1}-j}$. By Fact 1 of the previous section for some $i, \operatorname{nf}\left(\alpha_{i}\right)=\operatorname{nf}\left(\alpha_{i+1}\right)$ and so $\lambda^{\prime \prime} B_{1}^{m_{1}-i} \approx \lambda^{\prime \prime} B_{1}^{m_{1}-(i+1)}$. Therefore by congruence, $\lambda^{\prime \prime} B_{1}^{m_{1}-(i+1)} \approx$ $\lambda^{\prime \prime} B_{1}^{m_{1}}$ which is a contradiction. Next assume that $\alpha \stackrel{a}{\Longrightarrow}{ }_{n} \delta A_{1}^{n_{1}} \ldots A_{k}^{n_{k}}$ and $\beta A_{1}^{*} \ldots A_{k}^{*} \in \mathrm{E}(\alpha, a, n)$ and $\beta=\operatorname{nf}(\delta)$ and each $n_{i} \leq|\mathrm{E}(\alpha, a, n)|+1$. Because $\alpha \approx \gamma$ it follows that $\gamma \stackrel{a}{\Longrightarrow}{ }_{n} \gamma^{\prime}$ and $\beta A_{1}^{n_{1}} \ldots A_{k}^{n_{k}} \approx \gamma^{\prime}$. By Theorem 1 of section 3 either $\operatorname{nf}\left(\gamma^{\prime}\right) \in \mathrm{E}(\gamma, a, n)$ and therefore $\beta A_{1}^{n_{1}} \ldots A_{k}^{n_{k}} \approx \operatorname{nf}\left(\gamma^{\prime}\right)$, or $\gamma^{\prime}=\lambda B_{1}^{s_{1}} \ldots B_{m}^{s_{m}}$ and $\lambda^{\prime} B_{1}^{*} \ldots B_{m}^{*} \in \mathrm{E}(\gamma, a, n)$ and $\operatorname{nf}(\lambda)=\lambda^{\prime}$ and $\beta A_{1}^{n_{1}} \ldots A_{k}^{n_{k}} \approx \lambda^{\prime} B_{1}^{s_{1}} \ldots B_{m}^{s_{m}}$. We show that each $s_{i}$ can be chosen so that $s_{i} \leq \sum n_{i}+|\mathrm{E}(\gamma, a, n)|$. Let $\lambda^{\prime \prime}=\lambda^{\prime} B_{2}^{s_{2}} \ldots B_{m}^{s_{m}}$ and let $m_{1}$ be the smallest index such that $\beta A_{1}^{n_{1}} \ldots A_{k}^{n_{k}} \approx \lambda^{\prime \prime} B_{1}^{m_{1}}$. If $m_{1} \leq \sum n_{i}+|\mathrm{E}(\gamma, a, n)|$ then we let 
$s_{1}=m_{1}$ and repeat the argument for the other indices. Therefore assume that $m_{1}>\sum n_{i}+|\mathrm{E}(\gamma, a, n)|$. However $\lambda^{\prime \prime} B_{1}^{m_{1}} \stackrel{\epsilon}{\Longrightarrow} \lambda_{0} \lambda^{\prime \prime} B_{1}^{m_{1}-1} \stackrel{\epsilon}{\Longrightarrow}{ }_{0} \ldots \stackrel{\epsilon}{\Longrightarrow} \lambda_{0} \lambda^{\prime \prime} B_{1}^{0}$. Therefore let $\eta_{0}=\beta A_{1}^{n_{1}} \ldots A_{k}^{n_{k}}$, and so $\eta_{0} \stackrel{\epsilon}{{ }_{0}} \eta_{1} \stackrel{\epsilon}{\Longrightarrow}{ }_{0} \ldots \stackrel{\epsilon}{\Longrightarrow} \eta_{0} \eta_{m_{1}}$ and $\eta_{j} \approx \lambda^{\prime \prime} B_{1}^{m_{1}-j}$. Because $m_{1}>\sum n_{i}+|\mathrm{E}(\gamma, a, n)|$ it follows via fact 2 of the previous section that for some $i, \eta_{i} \approx \eta_{i+1}$ and so $\lambda^{\prime \prime} B_{1}^{m_{1}-(i+1)} \approx \lambda^{\prime \prime} B_{1}^{m_{1}-i}$ and therefore by congruence $\lambda^{\prime \prime} B_{1}^{m_{1}} \approx \lambda^{\prime \prime} B_{1}^{m_{1}-(i+1)}$ which contradicts that $m_{1}$ is a least index.

\section{References}

1. Baeten, J., Bergstra, J. and Klop, J. (1993). Decidability of bisimulation equivalence for processes generating context-free languages. Journal of Association for computing Machinery, 40, 653-682.

2. Caucal, D. (1996). On infinite transition graphs having a decidable monadic theory. Lecture Notes in Computer Science, 1099, 194-205.

3. Christensen, S. (1993). Decidability and Decomposition in Process Algebras. Ph.D thesis University of Edinburgh, Tech Report ECS-LFCS-93-278.

4. Christensen, S., Hirshfeld, Y. and Moller, F. (1993). Bisimulation equivalence is decidable for all basic parallel processes. Lecture Notes in Computer Science, $\mathbf{7 1 5}$, 143-157.

5. Christensen, S., Hüttel, H., and Stirling, C. (1995). Bisimulation equivalence is decidable for all context-free processes. Information and Computation, 121, 143148.

6. Esparza, J. (1997). Petri nets, commutative context-free grammars, and basic parallel processes. Fundamenta Informaticae, 30, 23-41.

7. Hirshfeld, Y. (1996). Bisimulation trees and the decidability of weak bisimilarity. Electronic Notes in Theoretical Computer Science, 5.

8. Hirshfeld, Y., Jerrum, M. and Moller, F. (1996). A polynomial-time algorithm for deciding equivalence of normed basic parallel processes. Journal of Mathematical Structures in Computer Science, 6, 251-259.

9. Hüttel, H. (1991). Silence is golden: branching bisimilarity is decidable for contextfree processes. Lecture Notes in Computer Science, 575, 2-12.

10. Hüttel, H., and Stirling, C. (1991). Actions speak louder than words: proving bisimilarity for context free processes. Proceedings 6th Annual Symposium on Logic in Computer Science, IEEE Computer Science Press, 376-386.

11. Jančar, P. (1995). Undecidability of bisimilarity for Petri nets and some related problems. Theoretical Computer Science, 148, 281-301.

12. Jančar, P., Kučera, A. and Mayr, R. (1998). Deciding bisimulation-like equivalences with finite-state processes. Lecture Notes in Computer Science, 1443, 200-211.

13. Milner, R. (1989) Communication and Concurrency. Prentice-Hall.

14. Sénizergues, G. (1998). Decidability of bisimulation equivalence for equational graphs of finite out-degree. Procs. IEEE FOCS'98, 120-129.

15. Stříbrná, J. (1999). Decidability and complexity of equivalences for simple process algebras. Ph.D thesis, University of Edinburgh, Tech. Report ECS-LFCS-99-408.

16. Stirling, C. (1998). Decidability of bisimulation equivalence for normed pushdown processes. Theoretical Computer Science, 195, 113-131. 